\title{
Energy Efficient Transmit-Receive Spatial Modulation for Uplink-Downlink Large-scale MIMO Systems
}

\author{
Ahmed Raafat ${ }^{1}$, Merve Yüzgeçcioğlu ${ }^{2}$, Mohammed Zahid Aslam ${ }^{3}$, \\ Adrian Agustin ${ }^{1}$, Josep Vidal ${ }^{1}$, Eduard Jorswieck ${ }^{2}$ and Yoann Corre ${ }^{3}$ \\ ${ }^{1}$ Dept. of Signal Theory and Communications, Universitat Politecnica de Catalunya, Barcelona, Spain \\ ${ }^{2}$ Communications Theory, Communications Laboratory, Technische Universität Dresden, Dresden, Germany \\ ${ }^{3}$ SIRADEL, Rennes, France \\ Email: \{ahmed.raafat, adrian.agustin, josep.vidal\}@upc.edu \\ $\{$ merve.yuzgeccioglu, eduard.jorswieck\}@tu-dresden.de, \{zaslam, ycorre\}@ siradel.com
}

\begin{abstract}
Energy efficient spatial modulation-aided uplink and downlink designs for future millimeterwave (mmWave) large-scale multiple-input multipleoutput (MIMO) systems are considered. Two novel uplink transceivers are proposed with the aim of considerably reducing the energy consumption at the user terminal, while achieving high spectral efficiency both in uplink and downlink transmissions. System performance is investigated using both stochastic and deterministic channels emulating real world urban scenarios.
\end{abstract}

I. INTRODUCTION

Energy efficient massive multiple-input-multipleoutput (mMIMO) implementation is having a significant impact in the design of the future wireless cellular networks as a result of the expected drastically increase in the number of devices. In particular, at millimeterwave (mmWave) band, the digital hardware, radiofrequency (RF) chains and analog-to-digital-converters (ADCs) are costly and power hungry [1].

Hybrid precoding schemes have been proposed to reduce the number of RF elements. However, hybrid architectures add many analog devices that could degrade the energy efficiency (EE) [2]. mMIMO systems based on 1-bit ADCs have been developed to improve the EE, however its application comes at the expenses of low spectral efficiency (SE) [3].

Recently, the spatial domain in mMIMO systems has been exploited with the purpose of simplifying the user terminal (UT) circuitry and also for attaining high SE. Conventionally, spatial modulation (SM) is employed at the transmitter side by mapping part of the information bits onto the transmit antennas. On the contrary, the spatially modulated symbols are mapped onto the receive antennas when receive spatial modulation (RSM) is considered. In [4], the authors developed downlink (DL) RSM scheme based on energy efficient UT architecture that attains high SE based on zeroforcing (ZF) precoder. In the case that the number of transmit antennas are much larger than the number of receive antennas, the linear precoding methods are sufficient and thus, ZF precoders are widely used in

The research leading to these results has received partly funding from the European Union's Horizon 2020 research and innovation programme under the Marie Skłodowska-Curie grant agreement No 641985, the project 5G\&B RUNNER-UPC (TEC2016-77148-C2-1R (AEI/FEDER, UE)) and the Catalan Government (2017 SGR 578AGAUR).
mMIMO systems because of the reasonable trade-off between complexity and performance. [5]. The authors in [6] show that RSM based on minimum-meansquare-error precoding achieves better performance under both total power constraint and per-antenna power constraints. The hybrid hardware architecture is combined with transmit spatial modulation (TSM) in [7]. In this work, the spatial domain is exploited by adopting antenna arrays instead of single antennas, with the goal of benefiting from beamforming gain.

In this paper, we exploit the spatial dimension at the UT to attain high SE in the UL and DL transmissions based on energy efficient UT's circuitry. Specifically, we propose two novel UL UT's architectures where each demands specific DL control signals overhead and hence the DL SE is affected by the UL transmission scheme selected. Thus, we design the UL architecture by taking special attention to the UL SE, DL SE, UL hardware power consumption and computational complexity. The contributions and novelties of this paper are as follows

- We propose energy efficient UL/DL spatial transmission schemes and propose a joint design.

- We develop two novel UL schemes based on novel energy efficient architectures that outperform the state-of-the-art in terms of SE and EE.

The rest of the paper is organized as follows. In Sec. II the general assumptions are introduced. In Sec. III and Sec. IV UL TSM and DL RSM architectures are depicted, respectively, and their performance are evaluated. In Sec. III-D the effect of the UL scheme on the DL SE is illustrated. A ray-based deterministic channel model has been utilized to validate the results in Sec. V. Finally, conclusions are provided in Sec. VI.

\section{SYSTEM MODEL}

Assume a point to point UL/DL mMIMO transmission scheme operating in narrowband and outdoor mmWave propagation environment. ${ }^{1}$ We consider energy efficient UT architecture where only one RF chain is equipped and a fully digital base station (BS). The

\footnotetext{
${ }^{1}$ The proposed scheme can be extended to wideband propagation by considering long symbols with multiple subcarriers (orthogonal frequency division multiplexing (OFDM)) or short symbols with single carrier and channel equalization.
} 


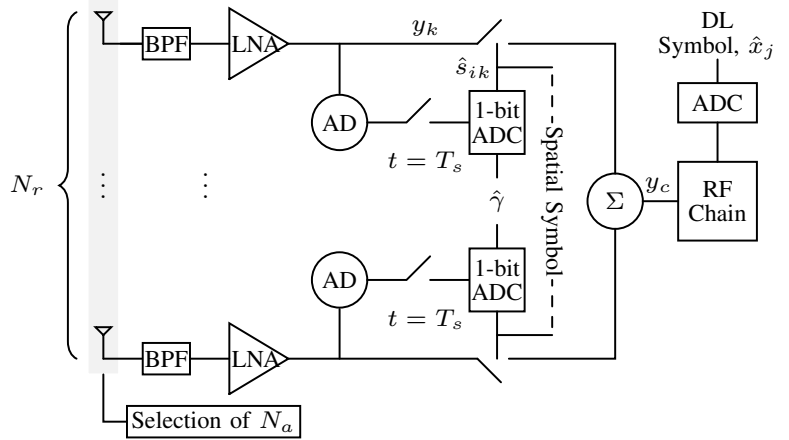

(a) Receive DL circuitry

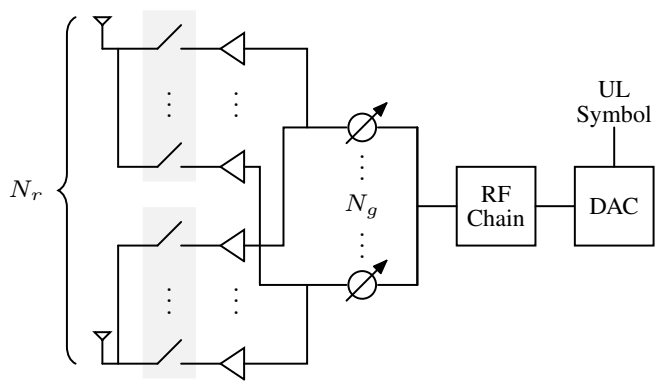

(c) Transmit UL circuitry - transmit antenna grouping

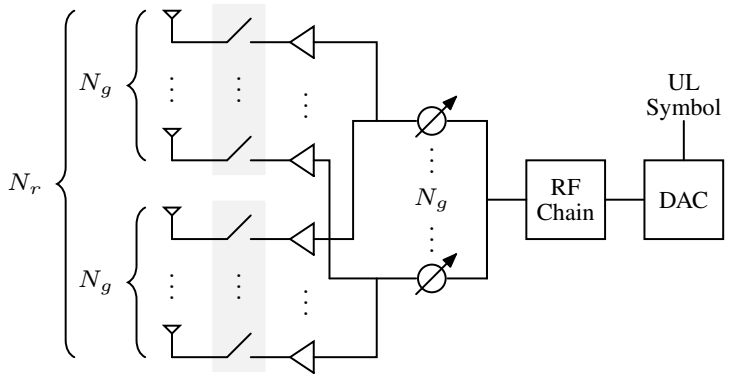

(b) Transmit UL circuitry - uniform grouping

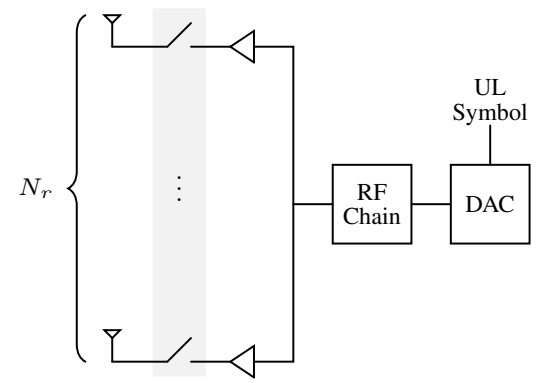

(d) Transmit UL circuitry - transmit antenna selection

Fig. 1: Low complexity UT circuitries

BS and the UT are equipped with $N_{t}$ transmit antennas and $N_{r}=N N_{g}$ UT antennas, respectively. Herein, $N$ is the number of groups and $N_{g}$ is the number of antennas at each group where the further details are given in Sec. III. The circuitry indicated in Fig. 1a is activated during the DL transmission which is designed to employ DL RSM detailed in Sec. IV. The circuitries indicated in Fig. 1b, 1c or 1d are activated during UL transmission that are designed in order to employ UL TSM detailed in Sec. III.

\section{A. Stochastic channel model}

The severe path-loss associated with mmWave propagation forces limited scattering environment and thus, we first utilize the stochastic geometry-based channel model [8] in evaluating the system performance where the channel matrix can be expressed as

$$
\mathbf{H}=\sqrt{\frac{N_{t} N_{r}}{\xi C}} \sum_{i=1}^{C} g_{i} \mathbf{v}_{r}\left(\theta_{i}\right) \mathbf{v}_{t}\left(\phi_{i}\right)^{H} .
$$

Herein, $\mathbf{H} \in \mathbb{C}^{N_{r} \times N_{t}}$ is the channel matrix with $C$ scattering clusters and path-loss $\xi . g_{i}$ is the $i^{\text {th }}$ path gain with $\mathcal{C N}(0,1)$ distribution, $\theta_{i}$ and $\phi_{i}$ are the azimuth angles of arrival and departure drawn from uniform distribution with $\mathcal{U}[-\pi / 2, \pi / 2]$ and $\mathcal{U}[-\pi / 6, \pi / 6]$ (directional transmission at the BS), respectively and $\mathbf{v}_{t}\left(\phi_{i}\right), \mathbf{v}_{r}\left(\theta_{i}\right)$ are the $i^{\text {th }}$ path transmit and receive array response vectors. We consider ULA where the $\mathrm{N}$-elements response vector can be expressed as

$$
\mathbf{v}(\phi)=\frac{1}{\sqrt{N}}\left[1, e^{j k d \sin (\phi)}, \ldots, e^{j(N-1) k d \sin (\phi)}\right]^{T},
$$

where $k=\frac{2 \pi}{\lambda}$ and $d$ is the inter-elements spacing.

\section{B. System assumptions}

We consider time-division-duplex (TDD) protocol where we exploit the channel reciprocity in developing transmission schemes such that the channel state information (CSI) is needed only at the BS. During the UL training, the BS can acquire the CSI by using, for example, a low complexity adaptive compress sensing based algorithm [9]. DL control signals are needed to enable the parameters of the DL detector (as explained in [4]) and UL transmission at the UT.

\section{UL Transmit Spatial Modulation}

In this section, we propose three UL transmission schemes based on three UL architectures as shown in Fig. 1b, 1c and 1d. We compare the three UL schemes in terms of SE, EE, computational complexity and the number of DL control symbols overhead.

\section{A. UL hybrid transmit spatial modulation}

In hybrid TSM as proposed in [7], the UT antennas are divided into $N$ groups each contains $N_{g}$ antennas. Incoming bit stream is divided into two parts where the first $\log _{2} N$ bits are used to choose the antenna array index and the remaining $\log _{2} M$ bits are modulated according to an $M$-ary modulation scheme. The UT chooses the analog beamformer for the selected antenna array and transmits the modulated symbol through this antenna array. We assume TDD reciprocity where the UL channel matrix $\mathbf{H}^{u}$ is the transpose of the DL channel matrix $\mathbf{H}$. The received symbol at the BS can be written as

$$
\mathbf{r}=\sqrt{P_{t}} \mathbf{H}_{n}^{u} \mathbf{f}_{n} x_{j}+\mathbf{n} .
$$

Therein, $x_{j}$ is the $j$-th symbol from $M$-ary constellation diagram, $\mathbf{f}_{n} \in \mathbb{C}^{N_{g} \times 1}$ is the analog beamformer vector of $n$-th antenna array and $\mathbf{H}_{n}^{u} \in \mathbb{C}^{N_{t} \times N_{g}}$ is 
the channel between $n$-th antenna array and the BS. Finally, $P_{t}$ is the average transmit power of the UT. We consider ULA analog beamformer where its response can be expressed as

$$
\mathbf{f}_{n}=\frac{1}{\sqrt{N_{g}}}\left[1, e^{j \pi \sin \left(\phi_{n}\right)}, \ldots, e^{j \pi\left(N_{g}-1\right) \sin \left(\phi_{n}\right)}\right]^{T},
$$

where $\phi_{n}$ is the quantized angle $\frac{2 \pi l}{L}$ from a codebook $\mathcal{F}=\left\{\mathbf{f}_{l} \in \mathbb{C}^{N_{g} \times 1}: \mathbf{f}_{l}^{H} \mathbf{f}_{l}=1, l=1, \ldots, L\right\}$ with $L=$ $2^{B}$ where $B$ represents the resolution of codebook.

The BS decides the best analog beamformer for each antenna array of the UT, so as to steer the power through the strongest path during the UL data transmission phase. This is done based on the acquired CSI as follows

$$
\mathbf{f}_{n}=\arg \max _{\forall \mathbf{f}_{l} \in \mathcal{F}}\left\|\mathbf{H}_{n}^{u} \mathbf{f}_{l}\right\|^{2} .
$$

The BS informs the UT about the best $f_{n}$ through DL control channel with low overhead as illustrated in the sequel. In the UL reception, the BS applies a digital combiner as follows

$$
\mathbf{y}^{u}=\mathbf{W} \sqrt{P_{t}} \mathbf{H}_{n}^{u} \mathbf{f}_{n} x_{j}+\mathbf{W n},
$$

where the digital combiner $\mathbf{W} \in \mathbb{C}^{N \times N_{t}}$ is constructed by the help of $\mathrm{ZF}$ principles as $\mathbf{W}=$ $\left[\mathbf{H}_{1}^{u} \mathbf{f}_{1}, \ldots, \mathbf{H}_{N}^{u} \mathbf{f}_{N}\right]^{\dagger}=\mathbf{D}^{\dagger}$. Herein, $\mathbf{y}^{u} \in \mathbb{C}^{N \times 1}$ contains the information of the selected UT antenna array index and the modulated symbol. Each entry of $\mathbf{y}^{u}$ has the following values

$$
\mathbf{y}^{u}(l)=\left\{\begin{array}{lll}
x_{j}+\mathbf{z}(l) & \text { if } & n=l \\
\mathbf{z}(l) & \text { if } & n \neq l
\end{array}\right.
$$

where $\mathbf{z}(l)$ is the $l$-th entry of $\mathbf{z}=\mathbf{W n}$. Finally, the transmitted symbol is decoded jointly by applying maximum-likelihood (ML) detector as

$$
\left[\hat{n}, \hat{x}_{j}\right]=\arg \min _{l, m}\left|\mathbf{y}^{u}(l)-\sqrt{P_{t}} x_{m}\right|^{2} .
$$

We evaluate the UL joint spatial and modulation bit error rate $P_{e}$ numerically by generating spatially modulated symbols, transmitting through the channel, detecting them and then calculating the number of correctly received bits per the total number of transmitted bits. Hence, we determine the UL SE $\left(R_{U L}\right)$ as

$$
R_{U L}=\left(\log _{2} N+\log _{2} M\right)\left(1-P_{e}\right)^{\log _{2} N+\log _{2} M},
$$

where $N$ and $M$ are designed to maximize the UL SE. A closed form expression of $P_{e}$ can be obtained from the union bound error probability of the ML detector.

\section{B. UL hybrid TSM with uniform grouping (UG)}

In this scheme, the UT antennas are uniformly distributed among the channel groups $\left(\mathbf{H}_{1}^{u}, \ldots, \mathbf{H}_{N}^{u}\right)$ where $\mathbf{H}_{1}^{u}$ and $\mathbf{H}_{N}^{u}$ include UT antennas indices from 1 to $N_{g}$ and $N_{g}(N-1)+1$ to $N N_{g}$, respectively. Mapping the UT antennas into groups does not depend on the channel and hence, UG does not require any computational complexity. The BS designs the quantized analog beamforming angles $\phi_{n}$ and informs the UT about them during the DL training. Thus,

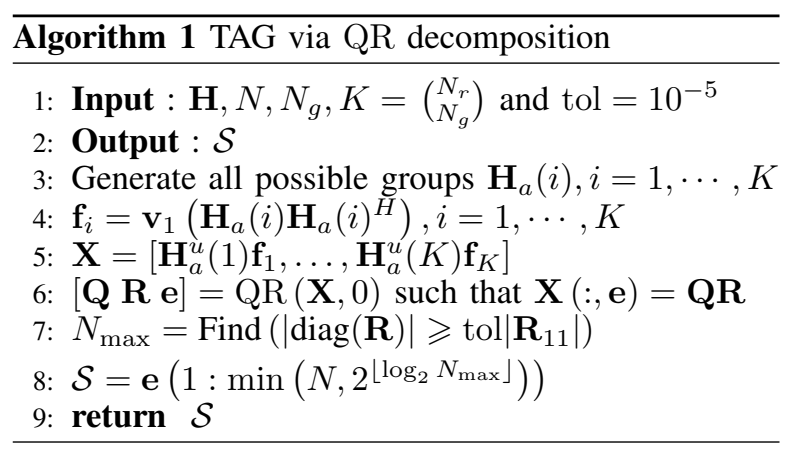

this scheme requires $N B / R_{D L}$ control symbols where $R_{D L}$ is the DL SE. Fig. $1 \mathrm{~b}$ shows that this scheme requires $N_{g}$ phase shifters and $N_{r}$ switches. However, UG could create correlation among the groups. Hence, the BS may be unable to distinguish between similar groups and as a result we could loose spatial data.

For the sake of improving the UL SE, we propose two novel UL SM schemes, each having specific hardware architecture and we compare their SE with the state-of-the-art (UG). Then, we show that the UL transmission scheme affects the DL SE due to the variation in the DL control signals overhead. Hence, the best UL scheme is designed based on UL SE, DL SE and UL EE.

\section{UL hybrid TSM with transmit antennas grouping (TAG)}

In this scheme, and for each channel realization, we select the best $N_{g}$ transmit antennas in each group to maximize the post processing received SNR. For simplicity, we consider infinite resolution codebook in selecting the best antennas inside each group where the analog beamformer can be expressed as

$$
\mathbf{f}_{n}=\mathbf{v}_{1}\left(\mathbf{H}_{n} \mathbf{H}_{n}^{H}\right)
$$

where $\mathbf{v}_{1}\{$.$\} denotes the maximum eigenvector. The$ associated post processing received $\mathrm{SNR}_{n}$ can be expressed as

$\mathrm{SNR}_{n}=\frac{P_{t} / \sigma^{2}}{\left[\mathbf{W} \mathbf{W}^{H}\right]_{n, n}}=\frac{P_{t} / \sigma^{2}}{\left[\mathbf{D}^{\dagger}\left(\mathbf{D}^{\dagger}\right)^{H}\right]_{n, n}}=\frac{P_{t} / \sigma^{2}}{\left[\mathbf{R}^{-2}\right]_{n, n}}$.

where $[\mathbf{Q} \mathbf{R} \mathbf{e}]=\mathrm{QR}(\mathbf{D}, 0), \mathbf{R}$ is a square upper triangular matrix and $\mathbf{D}(:, \mathbf{e})=\mathbf{Q R}$.

In Algorithm 1, we generate all possible channel groups and then, we select the best possible groups to maximize the received SNR shown in equation (11) through maximizing the diagonal entries of $\mathbf{R}$ in one step thanks to the $\mathrm{QR}$ decomposition. Although this scheme maximizes the received SNR, it demands $\left(\begin{array}{l}N_{r} \\ N_{g}\end{array}\right)$ maximum eigenvector computations and one QR decomposition that could be computationally complex for massive UT arrays. Moreover, extra DL control symbols are needed to inform the UT about the antennas selected in each group. This scheme requires $N\left(B+N_{r}\right) / R_{D L}$ control symbols. Fig. 1c shows that TAG needs $N_{g}$ phase shifters and $N_{g} N_{r}$ switches. 


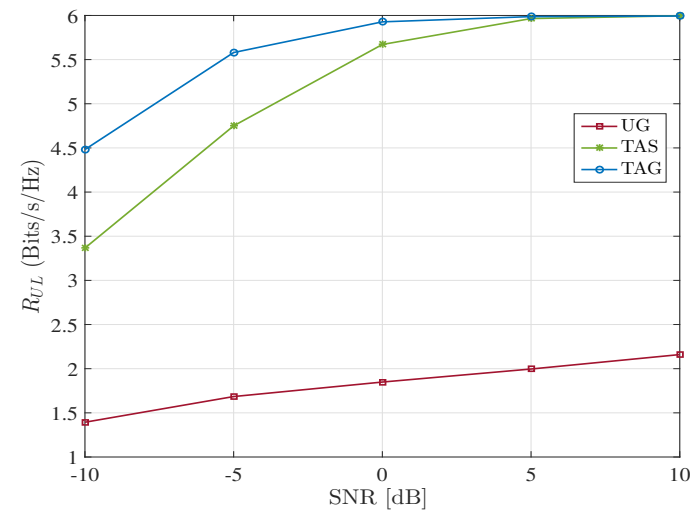

Fig. 2: UL SE of different UL transmission schemes at $N_{t}=128, N=4, N_{g}=4, C=3, \xi=1, \sigma^{2}=1$ and (average over 1000 realizations)

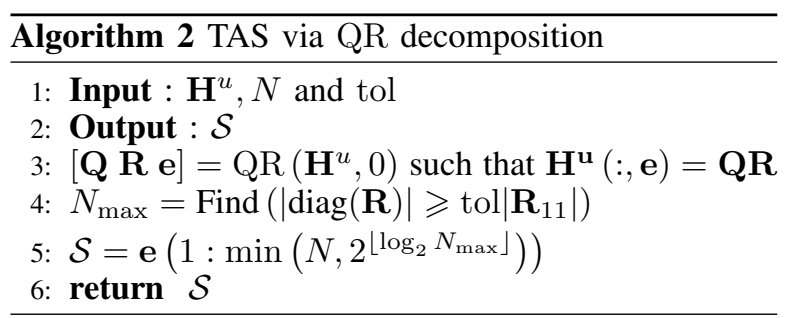

D. UL TSM with transmit antenna selection (TAS)

This scheme is a special case of TAG when each group contains one antenna without analog beamforming. The received SNR can be expressed as

$$
\mathrm{SNR}_{n}=\frac{P_{t}}{\sigma^{2}\left[\left(\left(\mathbf{H}^{u}\right)^{H} \mathbf{H}^{u}\right)^{-1}\right]_{n, n}} .
$$

In Algorithm 2, we select the best possible antennas to maximize the received SNR by applying the QR decomposition. This scheme requires only one $\mathrm{QR}$ decomposition. Therefore, it is much faster than TAG when $N_{g}$ is large and demands $\left(N B+N_{r}\right) / R_{D L}$ DL control symbols. As shown in Fig. 1d, the scheme requires $N_{r}$ switches and no phase shifters are needed.

\section{E. Impact of the selected UL scheme in the DL SE}

Assuming $N_{D L}$ is the total number of of DL channel uses per frame and $\frac{N_{r}}{R_{D L}}+1 \mathrm{DL}$ control channel uses are needed for all schemes to inform the UT about the DL active antennas and the DL detection threshold shown in Fig. 1a. Moreover, each UL architecture requires specific number of DL control symbols for the beamforing in the UL data transmission phase and thus the DL SE varies with the UL architecture as

$$
\begin{aligned}
\mathrm{SE}_{D L, \mathrm{UG}} & =\left(\frac{N_{D L}-\left(\frac{N_{r}+N B}{R_{D L}}+1\right)}{N_{D L}}\right) R_{D L}, \\
\mathrm{SE}_{D L, \mathrm{TAG}} & =\left(\frac{N_{D L}-\left(\frac{N_{r}+N B+N N_{r}}{R_{D L}}+1\right)}{N_{D L}}\right) R_{D L},
\end{aligned}
$$

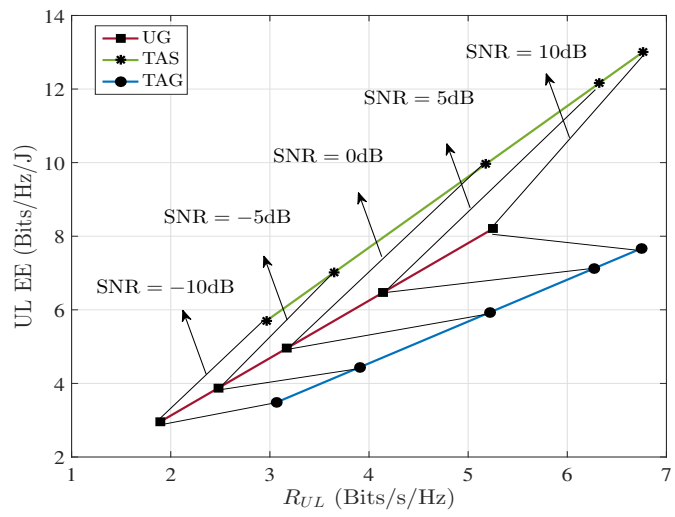

Fig. 3: UL SE vs EE of different UL transmission schemes at $N_{t}=128, N=4, N_{g}=4, C=5$, $\xi=1, \sigma^{2}=1$ and (average over 1000 realizations)

$$
\begin{aligned}
\mathrm{SE}_{D L, \mathrm{TAS}}=\left(\frac{N_{D L}-\left(\frac{2 N_{r}}{R_{D L}}+1\right)}{N_{D L}}\right) R_{D L} . \\
\text { F. } U L \text { circuitry power consumption }
\end{aligned}
$$

In this section, we apply a hardware power consumption model to evaluate the $\mathrm{EE}$ of the different UL architectures. The power consumption of different hardware components [2] can be expressed as

$$
\begin{gathered}
P_{\mathrm{PS}}=1.5 P_{\text {ref }}, P_{\mathrm{RF}}=2 P_{\text {ref }}, P_{\mathrm{DAC}}=10 P_{\text {ref }}, \\
P_{\mathrm{SW}}=0.25 P_{\mathrm{ref}}, P_{\mathrm{BB}}=10 P_{\mathrm{ref}}, P_{\mathrm{ref}}=20 \mathrm{~mW},
\end{gathered}
$$

where $P_{\mathrm{PS}}, P_{\mathrm{RF}}, P_{\mathrm{DAC}}, P_{\mathrm{SW}}$ and $P_{\mathrm{BB}}$ are the phase shifter, RF chain, digital-to-analog-converter, switch and base band power consumption, respectively, Hence, the power consumption of the different UL architectures can be expressed as

$$
\begin{gathered}
P_{c, \mathrm{UG}}=P_{\mathrm{RF}}+P_{\mathrm{DAC}}+N_{g} P_{\mathrm{PS}}+N_{r} P_{\mathrm{SW}}+P_{\mathrm{BB}}, \quad(18) \\
P_{c, \mathrm{TAG}}=P_{\mathrm{RF}}+P_{\mathrm{DAC}}+N_{g} P_{\mathrm{PS}}+N_{g} N_{r} P_{\mathrm{SW}}+P_{\mathrm{BB}}, \\
P_{c, \mathrm{TAS}}=P_{\mathrm{RF}}+P_{\mathrm{DAC}}+N_{r} P_{\mathrm{SW}}+P_{\mathrm{BB}} .
\end{gathered}
$$

Fig. 2 shows that TAG achieves the best performance due to its ability to combat the spatial correlation by optimizing the antennas inside each group. TAG outperforms TAS at low SNR due to the beamforing gain provided by TAG algorithm and TAS approaches the performance of TAG at high SNR.

Fig. 3 shows the SE-EE trade-off of the three UT architectures. TAS achieves superior EE, since it does not require phase shifters. The SE achieved by TAS at $C=5$ approaches the TAG. Hence, TAS is the best at sufficient SNR or at moderate scattering environment.

\section{DL Receive Spatial Modulation}

In the DL RSM, the BS transmits data per channel use in the form of two modulated symbols where $N_{a} \leqslant N_{r}$ data bits are mapped onto the spatial symbol $\mathbf{s}_{i} \in \mathbb{R}^{N_{a} \times 1}, i=1, \cdots, 2^{N_{a}}$ and $\log _{2} M$ data bits are mapped onto the $M$-ary constellation diagram modulation symbol $x_{j}$. The transmit symbol $\mathbf{x}_{i}^{j}$ at the precoder input can be expressed as

$$
\mathbf{x}_{i}^{j}=\left\{\begin{array}{lll}
\mathbf{s}_{i} x_{j} & \text { if } & \mathbf{s}_{i} \neq \mathbf{0}_{N_{r}} \\
\mathbf{s}_{i} & \text { if } & \mathbf{s}_{i}=\mathbf{0}_{N_{r}}
\end{array}\right.
$$




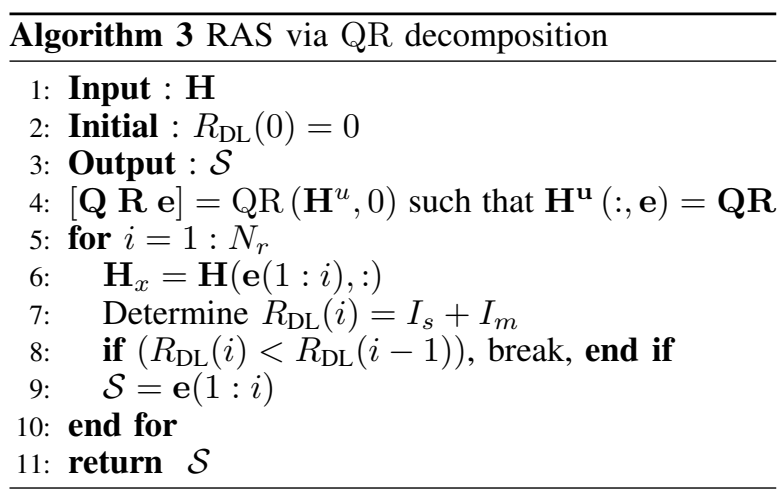

where the modulation symbol is not transmitted if the spatial symbol is all zeros. The symbol $\mathbf{x}_{i}^{j}$ passes through the precoder and the channel where the received symbol at the UT can be expressed as

$$
\mathbf{y}=\mathbf{H}_{a} \mathbf{P} \mathbf{x}_{i}^{j}+\mathbf{n} \text {. }
$$

Herein, $\mathbf{H}_{a} \in \mathbb{C}^{N_{a} \times N_{t}}$ is the channel after receive antennas selection (RAS) that illustrated in Sec. IV-B, $\mathbf{n} \in \mathbb{C}^{N_{a} \times 1}$ is the generated noise vector at the UT with independent and identically distributed (i.i.d.) circularly symmetric complex Gaussian entries, $\mathcal{C} \mathcal{N}\left(0, \sigma^{2}\right)$, and $\mathbf{P} \in \mathbb{C}^{N_{t} \times N_{a}}$ is a $\mathrm{ZF}$ precoder, fixed during the coherence time, and satisfies

$$
\mathrm{E}\left[\left\|\mathbf{P} \mathbf{x}_{i}^{j}\right\|_{2}^{2}\right]=\operatorname{Tr}\left\{\mathbf{P} \mathbf{R}_{s s} \mathbf{P}^{H}\right\}=P_{t},
$$

where $\mathbf{R}_{s s}=\mathrm{E}\left[\mathbf{s}_{i} \mathbf{s}_{i}^{H}\right]$ and $P_{t}$ is the transmit power. The ZF precoder can be expressed as

$$
\mathbf{P}=\sqrt{\alpha P_{t}} \mathbf{H}_{a}^{H}\left(\mathbf{H}_{a} \mathbf{H}_{a}^{H}\right)^{-1}
$$

where $\alpha=1 / \operatorname{Tr}\left\{\left(\mathbf{H}_{a} \mathbf{H}_{a}^{H}\right)^{-1}\right\}$ is a normalization factor used to fix the transmit power.

The detection of the spatial and modulation symbols for the DL RSM system based on ZF precoding is studied in [4]. In this system, each UT DL antenna is connected to an amplitude detector (AD) as shown in Fig. 1a. The signal measured by the $k^{\text {th }} \mathrm{AD}$ is compared with a threshold $\hat{\gamma}$ to detect the $k^{\text {th }}$ spatial bit such that $\hat{s}_{i k} \in\{0,1\}$. Then, the modulation symbol detection is performed on the combined signal

$$
y_{c}=\sum_{k=1}^{N_{a}} \sqrt{\alpha P_{t}} \hat{s}_{i k} s_{i k} x_{j}+\hat{s}_{i k} n_{k}
$$

through the RF chain to enable decoding of the modulation symbol $x_{j}$. Note that the received signal per antenna is allowed to pass through the RF chain only if the detected spatial bit is one $\left(s_{i k}=1\right)$. In this way noise is not enhanced.

\section{A. $D L S E\left(R_{D L}\right)$}

In [10], the authors show that the SE of the DL RSM scheme $R_{\mathrm{DL}}$ can be computed as spatial rate $I_{s}$ plus modulation rate $I_{m}$. As each DL UT antenna detects binary spatial bit, $I_{s}$ can be expressed using the binary asymmetric channel rate expression as

$$
I_{s}=N_{a}\left(\mathcal{H}\left(\frac{P_{1}+1-P_{0}}{2}\right)-\frac{\mathcal{H}\left(P_{1}\right)+\mathcal{H}\left(1-P_{0}\right)}{2}\right),
$$

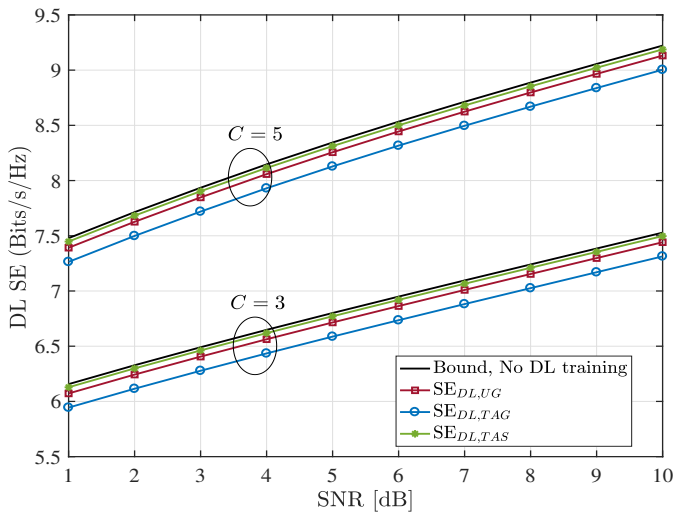

Fig. 4: DL SE corresponding to different UL transmission schemes at $N_{t}=128, N=4, N_{g}=4, \xi=1$, $\sigma^{2}=1$ and (average over 1000 realizations)

$$
\begin{gathered}
P_{1}=\operatorname{Pr}\left(\left|\sqrt{\alpha P_{t}}+n\right|>\hat{\gamma}\right)=Q_{1}\left(\frac{1}{\sigma} \sqrt{2 \alpha P_{t}}, \frac{1}{\sigma} \sqrt{2} \hat{\gamma}\right), \\
P_{0}=\operatorname{Pr}(|n|<\hat{\gamma})=1-Q_{1}\left(0, \frac{1}{\sigma} \sqrt{2} \hat{\gamma}\right)
\end{gathered}
$$

where $\hat{\gamma} \approx \frac{1}{2} \sqrt{\alpha P_{t}}, Q_{1}(x)$ is first order Marcum $\mathrm{Q}$ function and the entropy function $\mathcal{H}(P)=$ $-P \log _{2} P-(1-P) \log _{2}(1-P)$.

In [4], the authors showed that the constant amplitude constellation ( $M$-PSK) achieves the best performance for the DL RSM scheme, thus, we consider the shaping loss of the $M$-PSK symbols in evaluating the modulation symbol rate expression. Since we transmit one modulation symbol, $I_{m}$ can be computed using the multiple-input-single-output channel rate formula as

$$
\begin{gathered}
I_{m}=\sum_{i=1}^{2^{N_{a}}-1} \operatorname{Pr}\left(\mathbf{s}_{i}\right) \sum_{j=1}^{2^{N_{a}}} \operatorname{Pr}\left(\hat{\mathbf{s}}_{j} \mid \mathbf{s}_{i}\right) \times \frac{1}{2} \log _{2}\left(\left.\frac{4 \pi}{e} \mathrm{SNR}\right|_{\mathbf{s}_{\mathrm{i}}, \hat{\mathbf{s}}_{\mathrm{j}}}\right), \\
\left.\mathrm{SNR}\right|_{\mathbf{s}_{\mathrm{i}}, \hat{\mathbf{s}}_{\mathrm{j}}}=\frac{\left(\sum_{k=1}^{N_{a}} s_{i k} \hat{s}_{j k}\right)^{2}}{\max \left(\sum_{k=1}^{N_{a}} \hat{s}_{j k}, 1\right)} \frac{\alpha P_{t}}{\sigma^{2}}, \\
\operatorname{Pr}\left(\hat{\mathbf{s}}_{j} \mid \mathbf{s}_{i}\right)=\prod_{k=1}^{N_{a}} \operatorname{Pr}\left(\left|y_{i k}\right| \underset{\hat{s}_{j k}=1}{\gtrless} \hat{\gamma}\right), \operatorname{Pr}\left(\mathbf{s}_{i}\right)=\frac{1}{2^{N_{a}}} .
\end{gathered}
$$

\section{$B$. DL receive antenna selection}

The spatially sparse nature of mmWave propagation leads to badly conditioned and rank deficient channels if all receive antennas are considered and thus the $\mathrm{ZF}$ precoder is unfeasible. Channel full-rank can be ensured by smart selection of receive antennas. A possible criterion is the maximization of the DL SE. The RAS is performed at the BS and then the UT is informed through control channel. For the sake of reducing the computational complexity, we propose an efficient RAS algorithm based on QR decomposition a much faster approach than exhaustive search.

In Algorithm 3, we apply QR decomposition to sort the independent antennas in one step. Next, we select the receive antennas sequentially where we select one antenna per iteration and add it to the set $\mathcal{S}$ (the set 


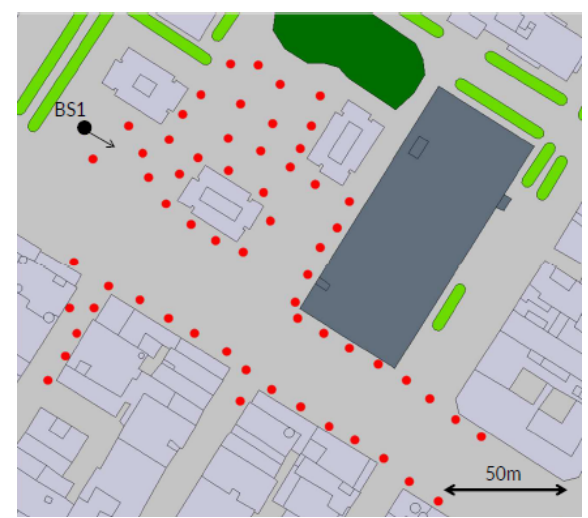

Fig. 5: Top view of realistic users distribution (red dots) inside mmWave small cell in Manhattan area in New York City.

includes the selected antennas). We stop adding more antennas when the DL SE decreases.

Fig. 4 shows the DL SE efficiency for the different UL architectures assuming $N_{D L}=500$ as in $5 \mathrm{G}$ New Radio. The DL SE is not sensitive to the UL architecture as the number of required DL control symbols for all architectures are much smaller than $N_{D L}$. Therefore, we select the best UL architecture based on UL SE, EE and the computational complexity.

\section{EVALUATION ON DETERMINISTIC RAY-BASED CHANNELS}

We validate the results in more realistic channel model assuming one 128 ULA BS and 60 user locations each with 32 ULA distributed inside a $28-\mathrm{GHz}$ small-cell in Manhattan area in New York City. 37 users are in line-of-sight connection with the BS as shown in Fig. 5. The antenna elements at the BS have $60^{\circ}$ aperture and the antenna elements at the MS are isotropic. The BS is 8 meter above the ground and the UTs are 1.5 meter above the ground. The mmWave mMIMO channels between the UTs and the BS are predicted from the ray-based Volcano technology by SIRADEL [11]. The Volcano model computes deterministic multiple paths that combine interactions with the buildings, trees and ground. It has been widely used in the last twenty years to assess the multi-path channel and network performance, either in urban, indoor and mixed environments. Afterwards, we compare the results obtained from the stochastic channel with the one obtained from the deterministic assuming the same large scale fading (path-loss $(\xi)$ computed from the deterministic simulator) at $\sigma^{2}=-84 \mathrm{dBm}$.

In Fig. 6, it can be seen that the DL SE based on the stochastic and deterministic channels are similar at $C=3$. We can clearly see the wide SE gap caused by the rich scattering environment when the number of clusters is $C=10$ that could enhance the spatial multiplexing and lead to unrealistic results.

\section{CONCLUSION}

In this work, a system model that employs RSM during DL transmission and TSM during UL transmission was studied. Three different UL circuitries with different complexity were introduced and their performance have been investigated in terms of SE and EE. Although each UL scheme demands different

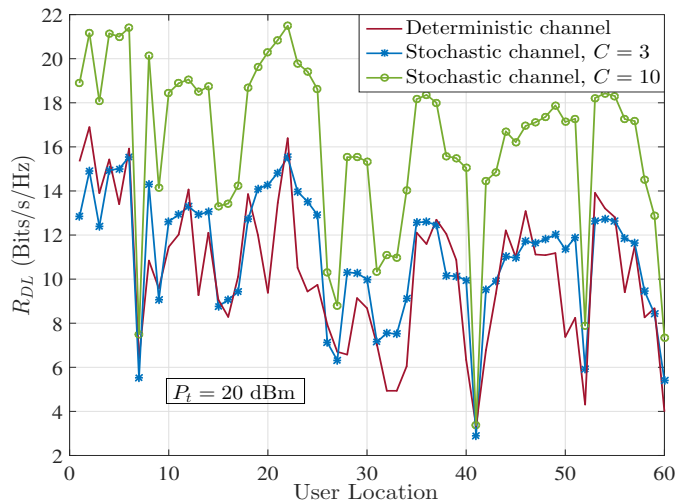

Fig. 6: DL SE vs users locations based on stochastic channels (average over 1000 realizations) and deterministic ray based channels at $N_{t}=128, N_{r}=32$, $C=\{3,10\}, P_{t}=20 \mathrm{dBm}$ and $\sigma^{2}=-84 \mathrm{dBm}$.

DL control signals overhead, the DL SE is not very sensitive to the selected UL architecture. TAS scheme has superior EE, less computational complexity and approaches the SE of TAG at sufficient SNR or moderate $C$ value. It has been shown that the urban scenario tends to have a low value of $\mathrm{C}$. To get more general conclusions, we need to extend the study to multi-cell scenario, evaluate the SE and EE of UL/DL schemes on deterministic channels and consider imperfect CSI.

\section{REFERENCES}

[1] F. Rusek and et al., "Scaling up MIMO: Opportunities and challenges with very large arrays," IEEE Signal Processing Magazine, vol. 30, no. 1, pp. 40-60, Jan. 2013.

[2] R. Méndez-Rial and et al., "Hybrid MIMO architectures for millimeter wave communications: Phase shifters or switches?," IEEE Access, vol. 4, pp. 247-267, Jan. 2016.

[3] J. Mo and R. W. Heath, "Capacity analysis of one-bit quantized MIMO systems with transmitter channel state information," IEEE Transactions on Signal Processing, vol. 63, no. 20, pp. 5498-5512, Oct. 2015.

[4] A. Raafat, A. Agustin, and J. Vidal, "Receive spatial modulation for massive MIMO systems," in IEEE Global Communications Conference (GLOBECOM), Dec. 2017.

[5] Thomas L. Marzetta, "Noncooperative cellular wireless with unlimited numbers of base station antennas," IEEE Transactions on Wireless Communications, vol. 9, no. 11, pp. 35903600, Nov. 2010.

[6] A. Raafat, A. Agustin, and J. Vidal, "MMSE precoding for receive spatial modulation in large MIMO systems," in IEEE International Workshop on Signal Processing Advances in Wireless Communications (SPAWC), June 2018.

[7] M. Yüzgeçcioglu and E. Jorswieck, "Hybrid beamforming with spatial modulation in multi-user massive MIMO mmwave networks," in IEEE Annual International Symposium on Personal, Indoor, and Mobile Radio Communications (PIMRC), Oct. 2017.

[8] M. R. Akdeniz et al., "Millimeter wave channel modeling and cellular capacity evaluation," IEEE Journal on Selected Areas in Communications, vol. 32, no. 6, pp. 1164-1179, June 2014.

[9] A. Alkhateeb and et al., "Channel estimation and hybrid precoding for millimeter wave cellular systems," IEEE Journal of Selected Topics in Signal Processing, vol. 8, no. 5, pp. 831846, Oct. 2014.

[10] A. Raafat, A. Agustin, and J. Vidal, "Receive antenna selection and hybrid precoding for receive spatial modulation in massive MIMO systems," in IEEE International Workshop on Smart Antennas (WSA), Mar. 2018.

[11] Y. Corre and Y. Lostanlen, "Three-dimensional urban EM wave propagation model for radio network planning and optimization over large areas," IEEE Transactions on Vehicular Technology, vol. 58, no. 7, pp. 3112-3123, Sept. 2009. 